\title{
Curved tails in polymerization-based bacterial motility
}

\author{
Andrew D. Rutenberg* \\ Department of Physics, Dalhousie University, Halifax, NS, Canada B3H $3 J 5$ \\ Martin Grant \\ Centre for the Physics of Materials, Physics Department, McGill University, 3600 rue University, Montréal, QC, Canada H3A 2T8
}

(Received 21 November 2000; published 19 July 2001)

\begin{abstract}
The curved actin "comet-tail" of the bacterium Listeria monocytogenes is a visually striking signature of actin polymerization-based motility. Similar actin tails are associated with Shigella flexneri, spotted-fever Rickettsiae, the Vaccinia virus, and vesicles and microspheres in related in vitro systems. We show that the torque required to produce the curvature in the tail can arise from randomly placed actin filaments pushing the bacterium or particle. We find that the curvature magnitude determines the number of actively pushing filaments, independent of viscosity and of the molecular details of force generation. The variation of the curvature with time can be used to infer the dynamics of actin filaments at the bacterial surface.
\end{abstract}

DOI: 10.1103/PhysRevE.64.021904

\section{INTRODUCTION}

The bacteria L. monocytogenes, S. flexneri, the spotted fever group of Rickettsiae, and the Vaccinia virus are intracellular pathogens that move through the continual polymerization of actin [1-4] in distinctively curved "comet tails" of actin filaments behind the motile particles. While fascinating on its own, the actin comet tail is functionally similar to the actin mesh in the lamellipodia of a locomoting eukaryotic cell, and the bacterial surface is analogous to the leading edge of the cell. Identification of the biochemical components involved has thus provided insight into the active regulation of actin polymerization by the cell $[5,6]$, an essential cellular process [7].

For polymerization-based motility, the force generated by actin polymerization at the moving object's surface drives the object forward against the viscous drag of the cytoplasm $[8,9]$. The necessary and sufficient bacterial contribution to motility is a single surface protein that orchestrates cellular cytoplasmic proteins to locally promote the nucleation, elongation, and cross-linking of actin filaments. In L. monocytogenes, this process is driven by the bacterial protein ActA [10-13], while $S$. flexneri expresses the protein IcsA for the same purpose $[14,15]$. Candidates for similar proteins have been proposed for spotted-fever Rickettsiae [3,16] and for the Vaccinia virus [17]. Simplified systems that have been developed for the study of polymerization-based motility include Escherichia coli expressing IcsA on their surfaces $[15,18]$ and microspheres coated with purified ActA [19]. Similar motility mechanisms appear to be at work in endosomal rocketing [20], and in nonactin polymerization-based motility systems derived from nematode sperm [21]. Actin polymerization-based motility may even play an important role in vesicle trafficking within the cell [22,23].

As the bacterium or particle is driven forward, a curved cometlike tail of actin filaments remains behind. Photobleaching experiments in L. monocytogenes [24] and qualitative observation of $S$. flexneri [15] and of spotted-fever

*Electronic address: http://www.physics.dal.ca/ adr/profile.html
PACS number(s): 87.16.Ka, 87.16.Ac, 87.17.Jj, 05.40.-a

Rickettsiae [3] demonstrate that the tail is stationary with respect to the surrounding environment, probably due to steric or functional connections with the cellular cytoskeleton, so that the shape of the tail represents the path of the bacterium. The curvature of this path varies from bacterium to bacterium, and changes over time for individual bacteria. It is not known what determines the bacterial path, and hence the tail curvature, though no active control or chemotactic behavior has been proposed for these systems.

Bacteria are functioning micromachines, but cannot be fully exploited without being fully understood. The L. monocytogenes motility system is understood well enough biochemically that ActA coated microspheres [19] should reconstitute polymerization-based motility in solutions of purified proteins [6], i.e., with total experimental control. However, we do not yet quantitatively understand the motility enough to be able to use the bacterial or microsphere motion as a probe of the bacterial or cellular conditions, or conversely to attempt to tailor those conditions to affect the bacterial motion.

In this paper, we propose that the curvature results from the random location of actin filaments pushing against the bacterium. We show how the curvature of the bacterial path can be used to predict static and dynamic structure at the bacterial surface. The average curvature is determined by the number of active filaments pushing the bacterium. Information about filament lifetime and surface diffusion rates may be obtained from curvature autocorrelations, since curvature depends on the location of active filaments with respect to the bacterial surface. If filaments are closely localized to specific bacterial surface proteins, then lifetimes and diffusivities of those proteins can be inferred. We focus on the most common experimental geometry of a thin quasi-twodimensional system constrained between a glass slide and cover slip, however we also discuss what would be expected for bulk geometries. In both cases, we discuss the apparent curvature appropriate to video microscopy.

\section{CURVED TRAJECTORIES}

Curvature is defined as the rate of rotation of direction, $K \equiv d \theta / d s$, or the rotation per unit path length, where $\theta$ is 


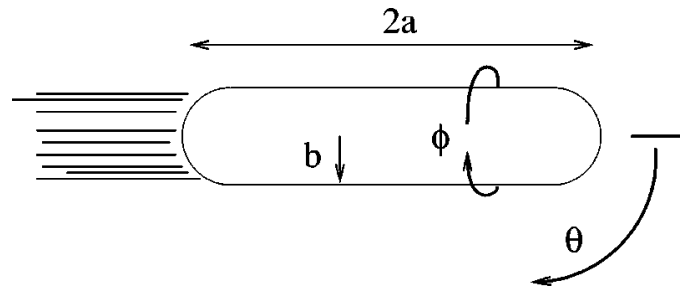

FIG. 1. The polar angle $\theta$ and the axial angle $\phi$ with respect to the capsule-shaped bacterium. The bacterium is taken to be curving within the plane of the page. The half-length and the radius of the bacterium are $a$ and $b$, respectively. A schematic actin comet-tail is shown to the left, and the bacterium is pushed towards the right. We approximate the bacterium as a prolate ellipsoid for purposes of drag calculations.

the polar angle in the current plane of motion (see Fig. 1) and $s$ is the distance measured along the particle path. The radius of curvature, $R \equiv 1 /|K|$, equals the radius of the circle that locally best fits the path. Either $K$ or $R$ locally characterize the path, i.e., both may vary along the bacterial path.

Curved trajectories imply that a torque $N$ is acting on the bacterium to balance viscous drag proportional to the angular rate of rotation, $N=\dot{\theta} C_{\text {turn }}$, where $C_{\text {turn }}=(32 \pi / 3) \eta\left(a^{4}\right.$ $\left.-b^{4}\right) /\left[\left(2 a^{2}-b^{2}\right) S-2 a\right]$ is a rotational drag coefficient for turning in $\theta$, and $\eta$ is the fluid viscosity, $a$ and $b$ are bacterial dimensions (see Fig. 1), and $S=2\left(a^{2}-b^{2}\right)^{-1 / 2} \ln \{[a$ $\left.\left.+\sqrt{\left(a^{2}-b^{2}\right)}\right] / b\right\}$ [25]. The viscous drag force $F$ due to linear motion at speed $v$ also carries a factor of the viscosity, where $F=v f_{\text {lin }}$ and $f_{\text {lin }}=16 \pi \eta\left(a^{2}-b^{2}\right) /\left[\left(2 a^{2}-b^{2}\right) S\right.$ $-2 a$ ] [25]. Since the curvature of the path, $K=\dot{\theta} / v$ $=\left(N / C_{\text {turn }}\right) /\left(F / f_{\text {lin }}\right)$, we can use the drag coefficients to obtain a remarkably simple result,

$$
K=\frac{3}{2\left(a^{2}+b^{2}\right)} \frac{N}{F},
$$

independent of the viscosity. This is fortunate since the effective viscosity of the cellular cytoplasm is strongly scaledependent, ranging from 0.01 poise $\left(10^{-3} \mathrm{Pas}\right)$ for small loops on dye molecules [26] to 2100 poise (210 $\mathrm{Pa} \mathrm{s}$ ) for $1.3 \mu \mathrm{m}$ diameter spheres [27]. We find that the curvature directly probes the ratio of force and torque applied to the bacterium, with an easily determined geometrical prefactor.

The force, $F=f_{0} n$, is proportional to the number of actively pushing filaments $n$, while the force per filament $f_{0}$ depends on the specific details of the motility mechanism, as seen explicitly in thermal-ratchet models of polymerizationbased motility [8]. A complementary coarse-grained elastic analysis of polymerization-based bacterial motion [9] exists, however it is not convenient for determining curvatures. We take $f_{0}$ as constant in time, which amounts to considering only times much greater than the mean time between actin monomer addition or equivalently distances much greater than the monomer size $2.7 \mathrm{~nm}$. This is appropriate, since the observed radii of curvature are larger than the bacterial scale (which is $1-2 \mu \mathrm{m}$ ).
To calculate the torque $N$, we must consider the torque due to each filament. These individual torques depend on exactly where the filament pushes on the bacterium. We assume that the $n$ actively pushing filaments are each randomly placed on the trailing end of the bacterium, so that each one will produce a random vectorial torque on the bacterium. The sum of many of these random torques will have a Gaussian distribution with zero mean. We can calculate the root-meansquare (RMS) torque, $N_{\text {rms }}$, from the local filament density. We take the growing filament barbed ends as uniformly distributed over the hemispherical cap at the end of the bacterium. In cross section, this leads to enhanced filament density at the edges of the tail, similar to that seen in thin-section electron micrographs of L. monocytogenes [28]. This distribution also follows naturally if the filament density follows a uniform ActA surface density. In cross-sectional coordinates, where the cylindrical radius $r$ ranges from 0 to $b$, the filament density distribution is

$$
P_{f}(r, \phi)=\sigma b / \sqrt{b^{2}-r^{2}},
$$

where $P_{f} r d r d \phi$ is the average number of filaments in the interval $(r, r+d r)$ and $(\phi, \phi+d \phi)$. Here, $\sigma=n /\left(2 \pi b^{2}\right)$ is the uniform surface filament density on the hemispherical end of the bacterium, and $b$ is the bacterial radius. The meansquare torque perpendicular to the direction of motion is easily found to be

$$
\left\langle N^{2}\right\rangle=f_{0}^{2} \int_{0}^{2 \pi} \int_{0}^{b} d \phi r d r\left[(r \sin \phi)^{2}+(r \cos \phi)^{2}\right] P_{f}(r, \phi),
$$

where $f_{0} r \sin \phi$ and $f_{0} r \cos \phi$ are the two components of the torque, so that they add in quadrature. This leads to an RMS torque $N_{\mathrm{rms}} \equiv \sqrt{\left\langle N^{2}\right\rangle}=f_{0} b^{2} \sqrt{4 \pi \sigma / 3}$. Taking the ratio $N_{\mathrm{rms}} / F$ to calculate the RMS curvature from Eq. (1), we have

$$
K_{\mathrm{rms}}=\frac{\sqrt{3}}{\left(a^{2}+b^{2}\right) \sqrt{4 \pi \sigma}}=\frac{b}{a^{2}+b^{2}} \sqrt{\frac{3}{2 n}}
$$

for a hemispherical distribution of filaments. Bacterial size and shape contribute to the curvature, as does the average distribution of filaments Eq. (2). Other surface distributions of filament densities are also possible, and would affect the geometric prefactors in Eq. (4) though not the functional dependence on the number of filaments $n$.

Remarkably, the average force per filament $f_{0}$ does not appear in our expression for the curvature, so that our results appear independent of the details of the force generation mechanism. However, within polymerization ratchet models [8], thermal fluctuations of semiflexible actin filaments transverse to their length [29] could generate transverse forces, which would lead to an increased torque and greater curvature than predicted above in Eq. (4). This transverse contribution would depend on the biomechanics of the coupling between the actin filament and the bacterial surface, which would also depend on the bacterial shape. Unfortunately it also depends on the effective and anisotropic elastic constants of the actin filaments [29], which in turn sensitively 
depend on how actively pushing actin filaments are crosslinked into the bacterial tail and the cytoskeleton, as can be seen by contrasting the elastic constants given by [29] and [30]. Our simplified treatment corresponds to no coupling of forces transverse to the direction of bacterial motion. We hope that sensitive experiments can uncover the effects of these transverse forces and hence yield more insight into polymerization-based force generation, though we expect the effects to show up predominately in the geometrical prefactor or amplitude of the curvature in Eq. (4) and not in the $1 / \sqrt{n}$ dependence. We expect our subsequent analysis, on distributions and autocorrelations of the curvature, to be unaffected.

The vectorial torque perpendicular to the bacterial direction of motion is Gaussian-distributed, and the curvature is proportional to the magnitude of the torque. Within a bulk [three-dimensional (3D)] geometry, the curvature has distribution

$$
P_{I}(x)=2 x e^{-x^{2}}
$$

where $x \equiv|K| / K_{\text {rms }}$ and $\int_{0}^{\infty} P_{I}(x) d x=1$.

\section{MEASURING CURVATURE}

The curvature of the bacterial path, characterized by Eq. (4) and Eq. (5), is intrinsic to a given random placement of $n$ filaments pushing against the bacterium. This intrinsic curvature is constant for fixed filament locations on the bacterial surface. At any given time, the intrinsic curvature represents circular motion around the curvature axis. However, the instantaneous curvature axis is not necessarily parallel to the line of sight, so the apparent path would appear elliptical and have a nonuniform curvature. We must also consider dynamical effects which change the direction of the curvature axis, both through rotation of the bacterium and through motion of the filaments on the bacterial surface.

Most experimental work to date has been done with restricted geometries, such as the typical gap of several microns [18] between a glass slide and its cover slip. For thin enough samples, curvature out of the plane will be restricted. If the axial angle $\phi$ measures the angle between the vectorial torque and the normal to the sample plane in the line of sight, then the apparent curvature as measured from a microscope or video image will be

$$
K_{\text {app }}=|K| \cos \phi
$$

Positive and negative curvatures correspond to clockwise and counterclockwise curved paths in the microscope image, respectively.

A single bacterium with a fixed intrinsic curvature, $K$, would eventually uniformly explore $\phi \in[0,2 \pi]$ through rotational diffusion [31]. As it does, the apparent curvature will change. For a fixed intrinsic curvature sampling at uniform time intervals, we would measure a distribution of apparent curvatures given by

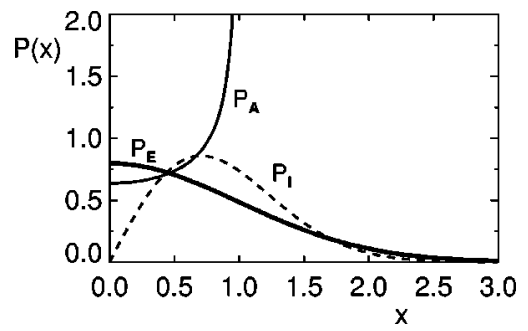

FIG. 2. The curvature distributions $P_{E}(x)$ (thick solid), $P_{A}(x)$ (solid), and $P_{I}(x)$ (dashed) for ensemble, apparent, and intrinsic curvatures, respectively, vs the scaled curvature, $x$ (see text). We expect $P_{E}(x)$ if a single bacterium is tracked over large times or if a collection of bacteria are tracked, $P_{A}(x)$ if enough data can be gathered for a single bacterium before the intrinsic curvature changes, and $P_{I}(x)$ for a single bacterium if the curvature is extracted from a sufficiently long $3 \mathrm{D}$ trajectory in a bulk sample.

$$
P_{A}(x)=\frac{2}{\pi \sqrt{1-x^{2}}}
$$

where $x \equiv\left|K_{\text {app }}\right| /|K| \in[0,1]$.

If an individual bacterium changes its intrinsic curvature in time, then over sufficient time $K$ will explore the entire intrinsic distribution, Eq. (5). The ensemble distribution $P_{E}(x)$ of apparent curvatures will then be given by Eq. (7) convoluted with Eq. (5):

$$
P_{E}(x)=\int_{x}^{\infty} d y P_{I}(y) P_{A}(y / x) / y=\sqrt{\frac{2}{\pi}} e^{-x^{2} / 2},
$$

where $x \equiv\left|K_{\text {app }}\right| /\left(K_{\mathrm{rms}} / \sqrt{2}\right)$, where the RMS-apparent torque is equal to $K_{\mathrm{rms}} / \sqrt{2}$. $P_{E}(x)$ also follows directly from the Gaussian distribution of each component of the torque. This "ensemble" distribution also characterizes the apparent curvatures of large groups of bacteria, since they will each have a different intrinsic curvature chosen from Eq. (5) and each will have a random axial angle $\phi$. The differences between the ensemble, apparent, and intrinsic curvature distributions are dramatic, as shown in Fig. 2.

For bacteria in a bulk sample, two angles are needed to characterize the bacterial path with respect to the viewer: a polar angle $\Psi$ with respect to the line of sight and an azimuthal angle $\phi_{0}$ about the line of sight. One must also specify the axial angle $\phi$ about the bacterial axis. If we line up the bacterium to travel away from us along the line of sight, before rotating it towards us by $\Psi$ in the $\phi_{0}$ azimuthal direction, and measure $\phi$ as the angle between the vectorial torque and $\phi_{0}$, then the apparent curvature is

$$
K_{\text {app }, 3 \mathrm{D}}=-|K| \cos \phi / \sin ^{2} \Psi .
$$

This is the curvature as measured from a microscope or video image, and takes values from $(-\infty, \infty)$ with no explicit $\phi_{0}$ dependence. Extremely large apparent curvatures are seen when bacteria are moving towards or away from the observer with correspondingly small velocities, with $\Psi$ small. The apparent speed is $v_{\text {app }}=v \sin \Psi$, where $v$ is the 
bacterial speed along its path. A convenient quantity is obtained by "normalizing" the apparent curvature by multiplying by $v_{\text {app }}^{2}$, to obtain

$$
\widetilde{K}_{\text {app }} \equiv K_{\text {app }, 3 \mathrm{D}} v_{\text {app }}^{2}=-\widetilde{K} \cos \phi,
$$

where $\widetilde{K} \equiv\left|K v^{2}\right|$. This also decreases the weight placed on apparently stationary bacteria, which can be hard to distinguish from other objects, and simplifies the analysis. $\widetilde{K}_{\text {app }}$ will have the ensemble distribution $P_{E}(x)$ if $\phi$ is uniformly explored in time. Note that time or path-length weighted distributions will differ in bulk samples, since the apparent speed $v_{\text {app }}$ will vary dramatically even for a constant intrinsic bacterial speed. The distributions presented in this paper are for time-weighted sampling, appropriate for video microscopy and/or for planar (2D) geometries.

\section{CURVATURE DYNAMICS}

For individual bacteria, the experimentally apparent variation of curvature from one moment to the next is striking $[3,18,32]$. Restricting ourselves to thin planar samples, the angular diffusion of the bacterial orientation can lead to changing apparent curvatures through changing axial angles $\phi$ in Eq. (10), and the intrinsic curvature can also vary if filament locations on the bacterial surface move significantly over time. We explore two cases: (i) where active (pushing) filaments are removed and randomly replaced on the bacterial surface, and (ii) where active filament locations randomly diffuse. We will later apply these cases to characterize filament repositioning for different motile systems.

It is easiest to characterize changes in the net torque acting on the bacterium with respect to a reference frame fixed to the bacterium. We consider the correlation of the intrinsic vector torques separated in time by $\Delta t>0$ :

$$
A_{N}(\Delta t) \equiv\langle\vec{N}(t) \cdot \vec{N}(t+\Delta t)\rangle=N_{\mathrm{RMS}}^{2} e^{-\Delta t / \tau},
$$

where a static intrinsic curvature corresponds to $\tau=\infty$, and the average is over the initial time $t$. The second line follows directly if each filament has a lifetime $\tau$ after which it is replaced randomly on the rear of the bacterium by another filament. If new filaments are randomly placed, they will be uncorrelated with other filaments. The correlation will then be proportional to the fraction of filaments that have not been replaced between the two times, i.e., $e^{-\left(t_{2}-t_{1}\right) / \tau}$. Exponential decay also applies for actin filaments whose fast-growing barbed-end positions diffuse over the bacterial surface with diffusion constant $D$. Solutions of diffusion on a spherical surface of radius $b$ [33] leads to $\tau=b^{2} /(2 D)$. These results would apply directly to proteolysis/replacement or surface motion of bacterial proteins such as ActA or IcsA if active filament positions are localized to such bacterial surface features (see below). They should also apply to dynamics intrinsic to the actin tail through capping and nucleation of active actin filaments [34], where capping is a loss mechanism. In this case, the time scale of autocorrelation decay would de- pend on the details of filament nucleation, namely how new filaments are placed with respect to preexisting active filaments.

Azimuthal diffusion will not affect the intrinsic curvature or its correlations, but it can contribute to decay of correlations of the apparent curvature by changing the apparent curvature over time. Azimuthal diffusion obeys $\left\langle(\Delta \phi)^{2}\right\rangle$ $=2 D_{A} \Delta t$, where $\Delta \phi$ is the net angle of rotation in the time $\Delta t$ and $D_{A}$ is the diffusion constant. This has a direct effect on the correlation between apparent curvatures separated in time by $\Delta t>0$ :

$$
\begin{aligned}
A_{\text {app }}(\Delta t) & \equiv\left\langle K_{\text {app }}(t) K_{\text {app }}(t+\Delta t)\right\rangle \\
& =\left\langle K_{\text {rms }}^{2}\right\rangle e^{-\Delta t / \tau}\langle\cos \phi(t) \cos (\phi(t)+\Delta \phi)\rangle \\
& =\left\langle K_{\text {rms }}^{2}\right\rangle e^{-\Delta t / \tau} e^{-D_{A} \Delta t} / 2,
\end{aligned}
$$

where $\tau$ is the decay time from intrinsic correlations in Eq. (11) and the average is over $t$ for a single bacterium. (We have used the identity $\left\langle e^{i x}\right\rangle=e^{-\left\langle x^{2}\right\rangle / 2}$ for Gaussiandistributed $x$.) Of course, rotation of the bacterium around its long axis will lead to decaying autocorrelations only if active filament tips are localized to bacterial surface features. If not, azimuthal diffusion might not affect the apparent curvature.

For individual bacteria tracked for times much less than $\tau$, the intrinsic curvature will appear constant. For times much longer than $\tau$, each bacterium will sample the ensemble of intrinsic curvatures. The characteristic time scale may be measured from the decay of curvature autocorrelations. Filament decay/replacement, filament diffusion, and axial rotation all contribute to exponential decay of the apparent curvature correlations. Their contributions to a particular motile system may be separated through independent measurements or through systematic studies where parameters such as the particle size or the cytoplasmic viscosity are varied.

Random rotation and diffusion of bacterial positions will also contribute to the measured curvature and curvature autocorrelations. In principle, this is a complicated hydrodynamic effect [35] leading to exponential asymptotic decay, however the time scales are very short $\left(\tau \sim R^{2} \rho / \eta\right.$ $\lesssim 10^{-5} \mathrm{~s}$ for a bacterium in a cell, where $R$ is the cell size and $\rho$ the cytoplasmic density) compared to the measurement intervals in typical bacterial experiments (seconds). The autocorrelation decay will effectively be discontinuous at $\Delta t$ $=0$, where thermal and measurement jitter will contribute at $\Delta t=0$ but not for $\Delta t>0$. To eliminate those contributions, the experimental RMS curvature $K_{\text {rms }}$ should be extracted from the $\Delta t \rightarrow 0^{+}$limit of the autocorrelations or should be fit from sufficiently long segments of the bacterial path.

Curvature autocorrelations in bulk samples are simple only when the intrinsic curvature is extracted from full 3D tracking of the bacterial trajectory (see, e.g., [36]). In that case, Eq. (11) will describe the autocorrelation decay.

\section{MOTILE SYSTEMS}

In this section, we discuss several specific motile systems, and use the details to refine our discussion of curved bacterial paths. For illustrative purposes, curvature has been esti- 
mated from published images of $L$. monocytogenes. This should be considered an order of magnitude estimate only. We only analyze motion within Xenopus laevis cell extracts, since cellular organelles and cell membranes, which can locally affect bacterial trajectories through collisions and which are hard to control for in published images, are absent. Also absent in extracts is a polarized cytoskeleton, which could plausibly align bacterial motion in intact cells; this could be explored through a systematic comparison of bacterial motion in cells and in cell extracts. It must be emphasized that proper studies of curvature require unbiased data and individual images previously selected for publication may be biased by aesthetic considerations. Distributions and autocorrelations require much more data than are available from published individual images, and will require analysis of video data.

The details of the nanoscale mechanical connection between the actin filament tail and a particular bacterium or motile particle are not yet known, nor are the details of the dynamics. Indeed, these details may differ for different bacteria or for different natural or reconstituted cytoplasmic environments. We present some plausible scenarios below and indicate the expected results of a curvature analysis in each.

\section{Listeria monocytogenes}

Motile L. monocytogenes have a distribution of tail lengths ranging up to about $15 \mu \mathrm{m}$ [28] and speeds of up to $0.4 \mu \mathrm{m} / \mathrm{s}$ [24]. Mature L. monocytogenes are roughly cylindrical Gram-positive bacteria, $1.5 \mu \mathrm{m}$ long with a diameter of approximately $0.5 \mu \mathrm{m}$ [32]. Polar surface expression of ActA is required $[10,11]$ for motility. L. monocytogenes in Xenopus extract [37] have apparent curvatures of approximately $K \approx 0.08 \mu \mathrm{m}^{-1}$, corresponding to $n=20$ filaments pushing on the bacterium. While this is a relatively small number, it is consistent with electron microscopy images [32].

The role of ActA in polymerization is being uncovered [38] but questions remain. It is not yet clear whether individual filaments are associated with individual ActA molecules, and if so, for how long. Direct mechanical attachment is possible and indeed indicated by optical tweezer studies [9]. Indirect attachment is also possible if the complex of ActA and cytoplasmic proteins serves as a source of monomeric actin with locally enhanced polymerization affinity. For example, profilin has been shown to interact with ActA through vasodilator-stimulated phosphoprotein (VASP) [11] and hence can provide a local "plume" of profilin-ATP-Gactin which in some conditions can polymerize more readily than ATP-G-actin [39]. We can readily obtain the leading behavior of the steady-state concentration $C$ diffusing a distance $d$ from a disklike source of radius $s$ and strength $C_{0}$ [40], $C / C_{0}=2 s /(\pi d)$. The diffusive plume would provide significantly enhanced polymerization only to distances on order the size $s$ of the ActA itself. If ActA is well separated on the bacterial surface, then filament nucleation and growth would be closely associated with individual ActA molecules, which in turn would be held stationary by the external peptidoglycan layer of the bacterium. The proteolysis induced lifetime $\tau$ of individual ActA is approximately $2 \mathrm{~h}$ in vivo [41], and would directly contribute to the curvature autocorrelation decay given by Eq. (12).

If there is a connection between the bacterium and its tail [9], then axial diffusion will be dramatically reduced and autocorrelation decay due to proteolysis should dominate and could be directly observed. However, if the bacterium is not tightly attached to its tail, then it will rotate with $D_{A}$ $=k_{B} T / C_{A} \approx 10^{-3} \operatorname{rad}^{2} \mathrm{~s}^{-1}$, corresponding to a time scale in Eq. (12) of $1 / D_{A} \approx 1000 \mathrm{~s} \ll \tau$, where $C_{A}=(32 \pi / 3) \eta\left(a^{2}\right.$ $\left.-b^{2}\right) b^{2} /\left(2 a-b^{2} S\right)$ is the viscous drag coefficient for axial rotation [25], and we take $\eta=30$ poise (3 Pa s) following [8].

\section{Shigella flexneri}

The Gram-negative S. flexneri has a similar motility mechanism to L. monocytogenes [15,42]; for example, it has a unipolar surface protein required for motility, IcsA. S. flexneri are about $2.3 \mu \mathrm{m}$ long and $0.5 \mu \mathrm{m}$ in diameter, and move at speeds comparable to L. monocytogenes [32]. However, differences are observed between $S$. flexneri and $L$. monocytogenes. The tails of $S$. flexneri appear to have fewer actin filaments than L. monocytogenes [43]. IcsA is targeted to one bacterial pole in $S$. flexneri and may diffuse in the outer membrane [44], in contrast to L. monocytogenes, where ActA is stationary.

Curvature studies can help investigate these differences. For example, if fewer filaments are actually pushing the bacterium, rather than simply fewer filaments involved in crosslinking, then according to Eq. (4), the curvature of the path of $S$. flexneri will be systematically larger. If actin filament tips were associated with individual IcsA, then diffusion of IcsA on the outer bacterial membrane would contribute a

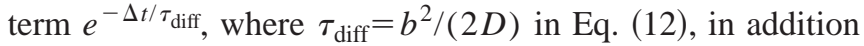
to the finite IcsA lifetime due to proteolysis [14]. Unfortunately, S. flexneri are not motile in Xenopus extracts $[18,32]$, but they do have qualitatively similar curvature to L. monocytogenes in intact cells [32].

\section{Spotted-fever Rickettsiae}

The spotted-fever group of Rickettsiae use actin-based motility for intracellular movement [16]. While a surface protein (rOmpA) of motile $R$. rickettsii has been implicated in tail formation and has sequence similarity to a domain of IcsA [45], its surface distribution and specific biochemical role have not yet been characterized. The most studied species, $R$. conorii [32] and $R$. rickettsii [3], are roughly the same size as $L$. monocytogenes, but move only one-third as fast.

While Rickettsiae are obligate pathogens that are not viable in Xenopus extracts, the curvature of their paths in intact cells is qualitatively smaller than that of L. monocytogenes or $S$. flexneri [32]. Yet the tails in $R$. conorii are found to have very long parallel filaments, with relatively few filaments observed in cross-section electron micrographs [32]. It is worth considering two possibilities. The first is that the filaments are not uniformly distributed on the bacterial surface. The numeric prefactor of the curvature Eq. (4) will range 
from 0 for polar filaments to $\sqrt{3 / 2}$ for uniform distribution on a hemisphere [Eq. (2)] to $\frac{3}{2}$ if all filaments are along the outer edge at $r=b$. A smaller than expected curvature can result from clustering of active filaments near the pole. However, this appears to be unlikely from the electron micrographs of decorated actin tails [32]. A second possibility is that the filaments are not randomly distributed on the bacterial surface. If the arrangement is symmetric, or regularly spaced, then the expected torque would be reduced. A paracrystalline surface structure is observed on Rickettsiae bacteria [46], though it is unknown whether it affects polymerization dynamics.

\section{VIRUSES AND VESICLES}

Actin polymerization-based motility is exhibited by the Vaccinia virus, where $\sim 200 \mathrm{~nm}$ diameter oblate virus particles move at $0.05 \mu \mathrm{m} / \mathrm{s}$ with tails of length $8 \mu \mathrm{m}$ in HeLa cells [4]. One of the interesting unsolved puzzles of this system is that the virus always travels in the symmetry direction, which is the orientation of highest drag.

If filaments are localized to the viral surface by a specific protein [17], and if it diffuses over the viral surface, the intrinsic curvature will change with time with $\tau=b^{2} /(2 D)$ following Eq. (11) and [33], where $b$ is the vesicular radius and $D$ is the diffusion constant of the motility protein on the viral membrane. Using $D \approx 10^{-9} \mathrm{~cm}^{2} / \mathrm{s}$, appropriate for diffusion within vesicular membranes, we have $\tau=0.05 \mathrm{~s}$. This is much less than the time scale of rotational diffusion and would dominate autocorrelation decay if present.

Polymerization-based motility with curved comet tails also occurs in motile vesicle systems, which are attractive systems for systematic study since they have fluid outer layers, spherical geometry, and a variety of sizes. Vesicle motion has been reconstituted in Xenopus egg extracts [22], in endocytosed vesicles [20], and in extracts of nematode sperm [21]. In some systems, the vesicle lipids directly mediate actin polymerization $[22,23]$, in which case the filaments are unlikely to be localized to particular spots on the vesicle surface. Effective filament motion could still occur due to random nucleation and loss of filaments from the vesicle surface, and this is a possible mechanism of intrinsic curvature autocorrelation decay in bacterial systems as well.

\section{MICROSPHERES}

A simplified in vitro system using small polystyrene microspheres, coated with purified ActA and added to Xenopus lavis egg extract, has been shown to reconstitute actin-based motility [19]. Could different intrinsic curvatures be sampled by a single "inert" microsphere? The ActA has its transmembrane domain replaced by a $6 \times$ His repeat, and is nonspecifically bound to the carboxylated polystyrene microsphere. It may be possible for the ActA to randomly crawl on the microsphere surface without detaching (for example, see [47]). Allowing a small surface diffusion rate, $D$ $=10^{-12} \mathrm{~cm}^{2} / \mathrm{s}$, results in a decay time for correlations of $\tau=b^{2} /(2 D) \simeq 325 \mathrm{~s}$ for a $0.5 \mu \mathrm{m}$ diameter microsphere. Data from curvature studies may thus may be able to dem- onstrate diffusion of ActA on the microsphere.

Microspheres are also good systems for systematic studies of various radii $b$ with a constant surface density of ActA and, presumably, actin filaments $\sigma$. In the autocorrelation decay, a finite filament lifetime would make a contribution that scales as $\tau \sim$ const, diffusion on the microsphere surface would have $\tau \sim b^{2}$, while axial diffusion would have $1 / D_{A}$ $\sim b^{3}$ [25]. Varying the cytoplasmic viscosity, on the other hand, should only affect axial diffusion, with $1 / D_{A} \sim \eta$.

\section{SUMMARY}

Random filament interaction with the bacterial or particle surface can explain the characteristic curved paths observed in polymerization-based motility systems, such as L. monocytogenes, S. flexneri, spotted-fever Rickettsiae, Vaccinia virus, and motile lipid vesicles and microspheres. We distinguish between the intrinsic curvature, which can only be measured with the full three-dimensional trajectory of the bacterium or particle, and the apparent curvature observed in microscope images. We derived explicit distributions for these curvatures and showed how they can uncover important qualitative differences between the various polymerization-based motility systems. We showed, in Eq. (12), how the lifetime and dynamics of surface-associated proteins, such as ActA or IcsA, affect the evolution of the intrinsic curvature of the motion of individual bacteria, virus particles, vesicles, or protein-coated microspheres.

Systematic experimental analysis of curvature in polymerization-based motility systems has not yet been done, but would supplement genetics, biochemistry, and microscopy by providing structural information about the interface between the actin tail and the bacterium. Curvature studies could estimate the number of filaments actively pushing the bacterium, the distribution of these active filaments on the bacterial surface, their localization with respect to motility protein complexes, and surface motility protein lifetime and diffusion on the bacterial surface. A similar analysis can be done for virus, vesicle, and microsphere systems.

It is useful to summarize the specific applications of this analysis. There are three. First, the relation between curvature and number of filaments in the actin tail, Eq. (4), can be compared with electron-microscopy cross sections and with normalized fluorescence studies. The relation between curvature and particle size, also in Eq. (4), can be used for different sized particles with similar surface preparations, such as microspheres. Second, the distribution of observed curvatures is predicted to be Gaussian, Eq. (8). For particles with constant intrinsic curvatures, a qualitatively distinct distribution of apparent curvatures, Eq. (7), is expected. If threedimensional tracking of particles in a thick sample is used, then the intrinsic distribution would apply, Eq. (5). Third, the variation of curvature in time is predicted to be described by an exponentially decaying autocorrelation function, Eq. (12). The time scale of autocorrelation decay, $\tau$, characterizes how the position of active filaments in the bacterial tail changes. Studies of different sized particles, or direct tracking of azimuthal particle rotation, could help to untangle the possible contributions to curvature autocorrelation decay. 
We have made simplifying assumptions to facilitate our analysis. We have assumed particular particle shapes and surface distributions of filaments. We also took the individual filament forces $f_{0}$ to be in the direction of particle travel. Different shapes, surface distributions, and filament orientations would change the numerical prefactor in Eq. (4), though the curvature distributions would not be affected. We have also assumed that individual filaments are independently randomly located. Nonrandom symmetric filament locations will result in curvatures much less than predicted in this paper. In contrast, filament distributions that are not symmetric will lead to qualitatively larger curvature than discussed here, where we assume a random but azimuthally symmetric distribution. We have also assumed that the viscosity does not vary strongly over bacterial length scales. Strong viscous heterogeneities, perhaps caused by particle motion itself, as well as local constraints posed by cellular organelles and membranes, will affect particle trajectories. This could dominate the effects described here in vivo.

\section{ACKNOWLEDGMENTS}

This work was supported financially by the Natural Sciences and Engineering Research Council of Canada, le Fonds pour la Formation de Chercheurs et l'Aide à la Recherche du Québec, and by the Canadian Institute of Advanced Research Program in the Science of Soft Surfaces and Interfaces. We would like to thank Lisa Cameron, Julie Theriot, and Bob Heinzen for discussions, and Sarah Keller and Jennifer Robbins for critical readings of earlier versions of the manuscript.
[1] J.A. Theriot, Annu. Rev. Cell Dev. Biol. 11, 213 (1995).

[2] S. Dramsi and P. Cossart, Annu. Rev. Cell Dev. Biol. 14, 137 (1998).

[3] R.A. Heinzen et al., Infect. Immun. 67, 4201 (1999).

[4] S. Cudmore et al., Nature (London) 378, 636 (1995).

[5] L.M. Machesky, Nat. Cell Biol. 1, E29 (1999).

[6] T.P. Loisel et al., Nature (London) 401, 613 (1999).

[7] B. Alberts et al., Molecular Biology of the Cell, 3rd ed. (Garland Publishing, New York, 1994).

[8] A. Mogilner and G. Oster, Biophys. J. 71, 3030 (1996).

[9] F. Gerbal et al., Pramana, J. Phys. 53, 155 (1999); Biophys. J. 79, 2259 (2000).

[10] C. Kocks et al., Cell 68, 521 (1992).

[11] G.A. Smith, D.A. Portnoy, and J.A. Theriot, Mol. Microbiol. 17, 945 (1995).

[12] E. Domann et al., EMBO J. 11, 1981 (1992).

[13] R.A. Brundage et al., Proc. Natl. Acad. Sci. U.S.A. 90, 11890 (1993).

[14] M.B. Goldberg et al., J. Bacteriol. 175, 2189 (1993).

[15] M.B. Goldberg and J.A. Theriot, Proc. Natl. Acad. Sci. U.S.A. 92, 6572 (1995).

[16] R.A. Heinzen et al., Infect. Immun. 61, 1926 (1993).

[17] F. Frischknecht et al., Nature (London) 401, 926 (1999).

[18] C. Kocks et al., Mol. Microbiol. 18, 413 (1995).

[19] L.A. Cameron et al., Proc. Natl. Acad. Sci. U.S.A. 96, 4908 (1999).

[20] C.J. Merrifield et al., Nat. Cell Biol. 1, 72 (1999).

[21] J.E. Italiano, Jr. et al., Cell 84, 105 (1996).

[22] L. Ma et al., J. Cell Biol. 140, 1125 (1998).

[23] A.L. Rozelle et al., Curr. Biol. 10, 311 (2000).

[24] J.A. Theriot et al., Nature (London) 357, 257 (1992).

[25] F. Perrin, J. Phys. Radium 5, 497 (1934).

[26] K. Luby-Phelps et al., Biophys. J. 65, 236 (1993).

[27] A.R. Bausch, W. Möller, and E. Sackmann, Biophys. J. 76, 573 (1999).
[28] L.G. Tilney, D.J. DeRosier, and M.S. Tilney, J. Cell Biol. 118, 71 (1992).

[29] K. Kroy and E. Frey, Phys. Rev. Lett. 77, 306 (1996).

[30] F.C. MacKintosh, J. Käs, and P.A. Janmey, Phys. Rev. Lett. 75, 4425 (1995).

[31] H. Berg, Random Walks in Biology, 2nd ed. (Princeton University Press, Princeton, NJ, 1993).

[32] E. Gouin et al., J. Cell. Sci. 112, 1697 (1999).

[33] D.E. Koppel, Biophys. J. 47, 337 (1985).

[34] L.M. Machesky and K.L. Gould, Curr. Opin. Cell Biol. 11, 117 (1999).

[35] I. Pagonabarraga et al., Phys. Rev. E 59, 4458 (1999); A.V. Zvelindovsky and A.V. Zatovsky, Nuovo Cimento D 19, 725 (1997).

[36] R. Thar, N. Blackburn, and M. Kühl, Appl. Environ. Microbiol. 66, 2238 (2000); P.D. Frymier et al., Proc. Natl. Acad. Sci. U.S.A. 92, 6195 (1995).

[37] J.A. Theriot et al., Cell 76, 505 (1994).

[38] M.D. Welch et al., Science 281, 105 (1998).

[39] D. Pantaloni and M.F. Carlier, Cell 75, 1007 (1993).

[40] J. Crank, The Mathematics of Diffusion, 2nd ed. (Oxford University Press, Oxford, 1975).

[41] M. A. Moors, V. Auerbach, and D. A. Portnoy, Cellular Microbiol. 1, 249 (1999).

[42] M.L. Bernardini et al., Proc. Natl. Acad. Sci. U.S.A. 86, 3867 (1989).

[43] W.L. Zeile, D.L. Purich, and F.S. Southwick, J. Cell Biol. 133, 49 (1996)

[44] J. Steinhauer et al., Mol. Microbiol. 32, 367 (1999).

[45] M. Charles et al., J. Bacteriol. 181, 869 (1999).

[46] E.L. Palmer et al., J. Bacteriol. 118, 1158 (1974); S.F. Hayes and W. Burgdorfer, Infect. Immun. 37, 779 (1982).

[47] E.J. Jervis, C.A. Haynes, and D.G. Kilburn, J. Biol. Chem. 272, 24016 (1997). 